Enf Neurol (Mex)

Vol. 12, No. 1: 48-52, 2013

CINNN, 2010

\section{Ensayo}

\title{
Filosofía, cronicidad y calidad de vida
}

\author{
Julio Hernández Falcón,* Adela Alba-Leonel **
}

\begin{abstract}
RESUMEN
Es necesaria la reflexión filosófica acerca de la cronicidad de los problemas actuales de salud y el concepto de bienestar. Lo filosófico, como materia de análisis, recupera el todo, rescata el mundo global, al mismo tiempo que el horizonte histórico del pensamiento occidental. La vida y la felicidad adquieren una morfología particular y cotidiana que aglutina tanto a los miembros de una comunidad como a un grupo dentro de un campo flexible pero limitado de opciones. Las realidades humanas, como las presentes en la enfermedad de carácter crónico, requieren definirse en relación con otra realidad, ya que la "mismidad" de cualquier cosa sólo es posible con una "otredad". El concepto de calidad de vida es tan controvertido que se ha considerado objeto de debate filosófico. Algunos autores sostienen que la calidad de vida se debe evaluar en términos de la utilidad, felicidad o satisfacción de deseos o preferencias. En la sociedad contemporánea, los conceptos como calidad de vida, bienestar y cronicidad demandan la reflexión de la enfermería de carácter filosófico hacia una búsqueda al encuentro consigo misma. Hoy por hoy, esto se estructura dentro del imaginario social atemporal y utópico, que no corresponde necesariamente a la intimidad necesaria del cuidado.
\end{abstract}

Palabras clave: Filosofía, cronicidad, calidad de vida, bienestar.

\section{Philosophy, chronicity and quality of life}

\begin{abstract}
Philosophical reflection is needed about the chronicity of the current problems of health and wellness concept. The unit of analysis is philosophical recovers everything recovers the global world while the historical horizon of western thought. Life and happiness in the way they acquire particular morphology welfare and everyday that brings both members of a community or a group within a flexible but limited field of life choices. Human realities as those present in a chronic disease requiring defined in relation to another reality as the "sameness" of anything is only possible with an "otherness". The concept of quality of life is so controversial, so it is considered an object of philosophical debate. Some authors philosophers argue that quality of life should be assessed in terms of utility, happiness or satisfaction of desires or preferences. In contemporary society, the quality of life concepts, wellness and chronic demand reflection of the nurse as a search philosophical required meeting with herself and one that today is structured within the social imaginary utopian timeless and not necessarily to privacy necessary care.
\end{abstract}

Key words: Philosophy, chronicity, quality of life, well-being.

\footnotetext{
* Lic. en Enfermería y Obstetricia, Maestro en Investigación de Servicios de salud, Profesor de Carrera Asociado “C”, Escuela Nacional de Enfermería y Obstetricia, UNAM.

** M. en C. en Epidemiología, Lic. en Enfermería y Obstetricia. Profesor de Carrera Asociado "C", Escuela Nacional de Enfermería y Obstetricia,
} UNAM.

Correspondencia: M. en C. Adela Alba-Leonel, Antiguo camino a Xochimilco y Viaducto Tlalpan, Col. San Lorenzo Huipulco, 14370, Tlalpan, México, D. F. Tel. 55562332 Ext. 239 E-mail: adelaalbaleonel@yahoo.com.mx

Este artículo puede ser consultado en versión completa en http://www.medigraphic.com/enfermerianeurologica 
$\mathrm{P}$ rácticamente, la sociedad actual está subordinada al lucro, y el individuo sólo le interesa en la medida en que rinda económicamente. Después de los 55 años de edad, la mayoría de las sociedades etiquetan a las personas como ancianos, lo cual los pone en un nivel de vida tan miserable que ambos conceptos, tanto viejo como pobre, son sinónimos. Es una paradoja que la vida moderna implique un empobrecimiento de este grupo etario.

La práctica médica y de enfermería hace necesaria la reflexión filosófica acerca de la cronicidad de los problemas actuales de salud y el concepto de bienestar. Lo filosófico, como unidad de análisis, recupera el todo; recupera el mundo global al mismo tiempo que el horizonte histórico del pensamiento occidental. ${ }^{1}$

En la antigua Grecia, la postura ante la vida distingue a las distintas escuelas: estoica, hedonista, escéptica y ecléctica. El hedonismo es el modo de vida más común entre la gente, cuyo ideal consiste en gozar al máximo los placeres, apartándose del dolor en la medida de lo posible; sus principales representantes son Epicuro y Lucrecio.

El estoico, en cambio, renuncia a los placeres y soporta los dolores y penalidades propias. De aquí la expresión: "soportó la pena en actitud estoica", así como la frase de Séneca: "Si accedes con agrado, el destino te llevará; si no, te arrastrará a la fuerza", por lo que los efectos displacenteros son sometidos al control y la decisión razonada. El estoicismo posee un enfoque racionalista puro sustentado en la impasibilidad (equilibrio completo) para hacer frente a las pasiones de la vida, de manera que el deber se asume a cualquier costo.

Por su parte, el escepticismo utiliza la duda como método y la aplica a todo; antes de aceptar alguna cosa, primero hay que demostrarla en sus causas. Esto justifica el saber escéptico como una duda encadenada.

El escéptico prefiere abstenerse de juzgar, oscila entre la afirmación y la no afirmación. Un escéptico no puede argumentar nada, pues con eso automáticamente se traicionaría. En el fondo, el escéptico tiene una oscura captación de lo absoluto, y ante la imperfección y contingencia de nuestras verdades se siente frustrado y opta por el camino más fácil: abstenerse de juzgar.

Para encontrar el sentido del ser y del bienestar es necesario entenderlo como la evaluación que resulta de una conciencia profunda, sensata y lúcida. No se trata de un bienestar basado en el placer corporal propio o de una conciencia primitiva o enferma, ya que el bienestar tiene componentes subjetivos y cognitivos, lo que nos brinda un componente individual de satisfacción por la vida.

El bienestar, según Zapf, ${ }^{2}$ incluye condiciones de vida buenas en términos objetivos y apreciación subjetiva de la vida. Cuando las condiciones de vida puntúan bien con medidas objetivas y la apreciación subjetiva de vida es positiva, se habla de "bienestar", mientras que cuando ambas son negativas se habla de "privación". Cuando la calidad objetiva es buena, pero la apreciación subjetiva es negativa, se aplica el término "disonancia", y la combinación de malas condiciones y buena apreciación se denomina "adaptación". Actualmente, la diversidad humana ha cobrado una enorme importancia donde existe una gran variedad de lógicas de sentido, las cuales nos permiten ver que no existe un solo modo de vivir sino muchos; y al mismo tiempo destacan la diversidad de los marcos de la realidad y necesidad que se afrontan. Como bien señala P. Bourdieu: "Para hablar de la fuerza de las formas que hace de las situaciones vitales - la naturaleza, la muerte, el sexo, la propia vida — una singular forma de ser y expresarse."3

La vida y la felicidad, en su forma de bienestar, adquieren una morfología particular y cotidiana que aglutina tanto a los miembros de una comunidad o a un grupo dentro de un campo flexible pero limitado de opciones de vida.

Octavi Fulat Genis ${ }^{4}$ puntualiza que cuando el hombre se sitúa en la postmodernidad se desentiende de cualquier trascendencia de Dios, hombre o razón, y se deslinda del mito y el rito. Incluso se ha desacralizado el llamado "orden del discurso" (Foucault), donde ya no se reflejan los valores, como es el caso de la bondad que se traduce en lo útil.

Muchas personas experimentan sensación de vértigo frente a los rápidos cambios del mapa geopolítico, del avance en la tecnología, la plasticidad del modelo de gestión de vida social y la crisis radical en los esquemas de pensamiento. Quienes viven bajo esta ficción "trófica" se encuentran frente a la desesperanza y la inseguridad y frente al anhelo por bienes inalcanzables, lo cual los lleva a la frustración. Diría Virilio: "Lo propio de la velocidad absoluta es también poder absoluto, control absoluto instantáneo, un poder casi divino... Esto ya no tiene nada que ver con la democracia, es pura tiranía." "Quien habla de poder, habla antes que nada de poder democrático y toda sociedad es sociedad de carrera." Al aludirnos, Virilio nos abarca a todos como especie planetaria, sujetos y objetos, una especie humana genérica. Su aseveración es contradictoria ya que el fenómeno de la transformación de la vida social no se puede considerar homogéneo sino jerárquicamente organizado y vivenciado y, por lo tanto, sujeto a una lógica de exclusión de los más desposeídos y del prójimo. La verdad globalizada es que los pobres han dejado de ser necesarios.

En el mundo contemporáneo, la transformación de los criterios de racionalidad, el reconocimiento de múltiples ópticas y formas discursivas se relaciona con la sensación de carencia informativa.

El propio quehacer de la enfermera se orienta hacia la idea de complejidad, integración, holismo y hacia las tradiciones 
y lógicas discursivas, descartando otras posibilidades de lectura. La propia tradición humanista cristiana ha sido tocada en su representación y en su aportación al cuidado, ya que se hunde en lo profundo del quehacer racional y técnico.

La enfermedad crónica es "una condición física o mental que requiere largos periodos (mayores de seis meses) de monitoreo y/o manejo para controlar los síntomas y detener el curso de la enfermedad". Evidentemente, el cuidado en estas circunstancias implica un gran esfuerzo, y el cuidador requiere apoyo de los miembros de la familia, el grupo social y el personal de salud, incluyendo a la enfermera. Es frecuente que la situación económica sea difícil por la necesidad de medicamentos y otros tipos de tratamiento, por lo que se requiere de respuestas sociales organizadas que atenuen la situación.

La propia Organización Mundial de la Salud define a las enfermedades crónicas como "enfermedades de larga duración y por lo general de progresión lenta"; se trata de las principales causas de muerte en el mundo y son responsables del 63\% de todas las muertes (2012). ${ }^{6}$ Las enfermedades crónicas ponen en evidencia el conflicto entre la enfermedad y la satisfacción terapéutica, e incluso generan un movimiento alternativo de búsqueda de medicina alternativa, y de un creciente mercado de terapeutas.

Las realidades humanas como las presentes en la enfermedad de carácter crónico requieren definirse en relación con otra realidad ya que la "mismidad" de cualquier cosa sólo es posible con una "otredad". Bajo esta situación, E. Levinas señala que "el criterio de identidad significa una relación asimétrica entre el yo y el tú, entre el yo y el otro". Este mecanismo es una prioridad subjetiva y existencial de "mi ser". Martín Buber sostiene que el "yo" adquiere conciencia de su conexión y su separación, de cómo relacionarse con el mundo para distinguirse de él y autoidentificarse.

Levinas nos dice que "la relación de lo mismo con el otro no es dialéctica sino una posibilidad de la metafísica que traduce lo concreto del egoísmo". La experiencia humana de la enfermera es consustancial con la relación establecida con el otro y con el hecho de vivir y existir, pero sobre todo, de tomar conciencia. La conciencia subjetiva deja de ser anterior a cualquier proceso de relación con el mundo para constituirse en alteridad vulnerada por el otro. ${ }^{7}$

Desde la perspectiva de los cuerpos, la cronicidad de la enfermedad y el bienestar humano asumen la vocación de autoconocimiento que distingue el quehacer filosófico. En todo sentido, el cuerpo es la base de todas las referencias cognoscitivas. Temporalidad y corporeidad están sólidamente unidas y más aún las producciones son la extensión del propio cuerpo humano. No existe, de esta forma, expresión cultural que no se remita al cuerpo. Sin embargo, la experiencia histórica del propio cuerpo asume diferencias que tienen la conciencia reflexiva y la disposición para interpretar el cuerpo y el entorno. Cada sujeto recibe a lo largo de su vida una compleja red de elementos de juicio; éstos son conscientes e inconscientes, físicos y mentales, para normar su conducta en relación del cuerpo propio y de los semejantes. Según Jean Paul Sartre, el ser del hombre es un "estar ahí", un estar situado de manera contingente, en algún lugar y en relación con las cosas y las personas. Este "estar ahí" se manifiesta como el cuerpo.

El intercambio entre cuerpos corresponde con la modificación del espacio y el tiempo psíquicos. Nuestras impresiones sensoriales lo testifican ante un estado de ánimo o salud, por lo que las impresiones cambian. Quien está en situación de enfermedad se encuentra en la espera prolongada y en situación de bienestar de la experiencia de una duración limitada.

La descripción más común de calidad de vida es la ausencia de defectos funcionales en el cuerpo, una función corporal inalterada. Los psicólogos la definen como la ausencia de defectos mentales. La buena calidad del cuerpo y la mente trabajan de acuerdo a su propósito.

Aristóteles aporta el concepto de eudaimonía, cercano a la actividad del concepto de calidad de vida. Finalmente, el arte de vivir denota habilidades especiales de vivir y disfrutar de la vida.

\section{CALIDAD DE VIDA}

El concepto de calidad de vida es tan controvertido que se ha considerado objeto de debate filosófico. Algunos autores filósofos sostienen que la calidad de vida se debe evaluar en términos de la utilidad, felicidad o satisfacción de deseos o preferencias. El gran doctor en felicidad, Schopenhauer, dividió las necesidades humanas en tres clases: las naturales y necesarias, que al no ser satisfechas producen dolor (dentro de éstas se encuentran el alimento y el vestido, relativamente fáciles de satisfacer); las naturales pero no necesarias, como la satisfacción sexual, más difíciles de satisfacer; y las que no son naturales ni necesarias, como el lujo, la abundancia, el protagonismo y el esplendor, siendo su satisfacción más difícil. ${ }^{8}$

Durante varios siglos, el término "felicidad" se ha utilizado para todos los significados de calidad de vida. Sin embargo, en filosofía permanecieron los siguientes significados: En filosofía social, el significado de "buenas condiciones de vida" (felicidad como buena sociedad) y filosofía moral, el significado de buenas acciones (felicidad como virtud). En las ciencias sociales prevaleció la palabra "felicidad" como disfrute subjetivo de la vida. ${ }^{9}$

La vida implica en una persona una combinación de seres y quehaceres, los que denominamos funcionamientos, 
siendo éstos las diferentes facetas en las que un ser humano puede tener interés o bien se puede ver realizado (salud, alimentación, educación, trabajo, diversión, placer, política, relaciones, entre otros).

La calidad de vida está íntimamente relacionada con las capacidades que tiene una persona, entendiéndose capacidad como la libertad del ser humano para elegir o seleccionar la clase de vida que quiere para sí mismo. ${ }^{10}$

Nussbaum propone, desde la perspectiva aristotélica, determinar "una lista de funcionamientos que constituyen una buena vida humana", como ciertas características de humanidad, aunque se experimenten de manera diferente en las distintas culturas: la mortalidad, el cuerpo humano, la capacidad de placer y dolor; la capacidad cognitiva, la razón práctica, el desarrollo infantil temprano, la afiliación o sociabilidad, el humor y sentido lúdico. De entre ellas, hay dos, la afiliación (o sociabilidad) y la razón práctica, que desempeñan, según Nusssbaum, un papel arquitectónico en la vida humana al permear y organizar las demás funciones, determinando lo que deba entenderse como "naturaleza humana"."

Una persona se refiere a la libertad sustantiva para conseguir distintas combinaciones de funciones, esto es, para lograr el estilo de vida que uno quiere. Lo primordial no son las funciones (las cosas que se hacen), sino las capacidades (las oportunidades reales). ${ }^{12}$

La calidad de vida puede ser un ideal objeto de búsqueda continua por parte del ser humano que simultáneamente se encuentra en permanente cambio, puesto que representa un equilibrio dinámico entre lo que se es, lo que se tiene y lo que uno representa individualmente y como ser gregario. ${ }^{7}$ Se debe tener una concepción de un ser humano integral en el que confluyan lo biológico, lo psicológico, lo social, lo ético, lo político, lo cultural, lo histórico, lo económico, lo espiritual y lo estético, sin que a priori ningún aspecto sea más relevante que otro para determinar la calidad de vida; sin embargo, para algunos la clave puede estar en lo económico, siendo que otros consideran que lo cardinal está en el ser y el hacer, esa capacidad de elegir que postulan Nussbaum y $\operatorname{Sen}^{9}$ que permite el logro de buenos fines y una vida buena y floreciente. Es decir, la integración de la reflexión y la acción de la contemplación y el darle sentido a la vida en vista de que al entender el porqué de algo es más fácil hacer frente a los cómo, empleando la filosofía como terapia y reivindicando la máxima de Nietzsche: "Podremos manejar cualquier cómo, si tan sólo tenemos un por qué"; 13 siempre que esos cómo y por qué tengan sentido constructivo y edificante para la propia vida y para la de los demás. Nussbaum busca que los ciudadanos vivan bien y que desarrollen ciertas capacidades para llevar una vida plena, ${ }^{14,15}$ por lo que plantea diez capacidades funcionales humanas bási- cas que las sociedades deberían brindar a sus ciudadanos; éstas son: ser capaz de vivir hasta el fin de una vida humana completa, tanto como sea posible, no morir prematuramente o antes de que esté tan reducida que no valga la pena vivirla; ser capaz de tener buena salud, estar adecuadamente nutrido, tener la protección necesaria, tener oportunidades para la satisfacción sexual; ser capaz de evitar el dolor innecesario y no beneficioso y tener experiencias placenteras; ser capaz de usar los cinco sentidos, de imaginar, pensar y razonar; ser capaz de ligarse a cosas y otras personas, amar a aquellos que nos aman y cuidan, sufrir frente a su ausencia, sentir gratitud y amor; ser capaz de formar un conceto del bien y tener una reflexión crítica sobre la planificación de la propia vida; ser capaz de vivir para y con otros, reconocer y mostrar preocupación por otros seres humanos, involucrarse en interacciones familiares y sociales; ser capaz de vivir en relación con el resto del mundo natural; ser capaz de reír, jugar y disfrutar de actividades recreativas; y ser capaz de vivir la propia vida en el propio contexto.

El enfoque de las capacidades coloca las decisiones acerca de una vida mejor del lado de los individuos, es decir, la calidad de vida no depende de decisiones colectivas. No así las condiciones de vida, que obviamente han de ser colectivas, tanto si las decisiones vienen del Estado o como demandas de la sociedad civil. ${ }^{16}$

Actualmente, existen tópicos que se consideran sustantivos para evaluar una vida como poseedora de calidad, o bien tendencias objetivas, fundamentalmente la satisfacción de aspectos básicos como lo planteó Maslow en su pirámide. ${ }^{17}$ Así mismo, otros autores proponen un modelo de calidad de vida, considerando a priori una cobertura de necesidades básicas para la supervivencia, por lo que conciben al ser humano inmerso en la sociedad, enmarcada en un lugar y una cultura, elementos que regulan e incluso limitan la cosmovisión del sujeto. ${ }^{18}$

\section{CONSIDERACIONES}

En la sociedad contemporánea, los conceptos calidad de vida, bienestar y cronicidad demandan la reflexión de la enfermera de carácter filosófico para realizar una búsqueda tanto en sí misma como en los otros. Hoy por hoy, esto se estructura dentro del imaginario social atemporal y utópico que no corresponde necesariamente a la intimidad necesaria del cuidado. El carácter complejo de los cambios globales que expresa el cuidado ampara un criterio cultural una vez que sintetiza su naturaleza desde la perspectiva holística. Los nuevos escenarios del imaginario social sugieren la necesidad de redefinir al sujeto en el contexto actual, juzgando tanto la dimensión individual como la social. El empoderamiento de la enfermera es una actualización que 
debe afrontar tanto el nuevo concepto de sujeto como de los efectos de la homogeneización y heterogeneidad de la cultura.

Los nuevos pactos con los sujetos de atención y un redescubrimiento de las posibilidades de la propia enfermedad crónica parecen sin duda uno de los elementos más importantes del futuro de la salud y el cuidado.

\section{BIBLIOGRAFÍA}

1. Ayestarán I, Insausti X, Águilar R. Filosofía en un mundo global. Barcelona: Ed. Ánthropos; 2008.

2. Zapf W, Glatzer W. German social report: living conditions and subjective well-being (1978-1984). Social Indicators Research. 1984; 25.

3. León-Vega E, Serna-Arango J, Mejia-Rivera O. Sentido ajeno. México: Ed. Anthropos; 2005: 29-34.

4. Fulat GO. Axiológica educativa para Occidente.

5. Op. Cit. León-Vega E. Sentido ajeno. 2005: 19 y 20.

6. OMS. Enfermedades crónicas [internet]. [acceso 13 de noviembre 2012]. Disponible en http://www.who.int/topics/chronic_diseases/es/

7. León Vega E. Sentido ajeno. México: Ed. Anthropos; 2005: 30-39.

8. Schopenhauer A. Arte del buen vivir. 19a Edición. Madrid: Editorial EDAF, S.A; 2006.

9. Ovalle O, Martínez J. La calidad de vida y la felicidad. Escuela de Economía Internacional, UACH. En: Contribuciones a la Economía
[Internet]. 2006. Disponible en: http://www.eumed. net/ce/2006/oojm. htm

10. Nussbaum M, Sen A. La Calidad de Vida. México: Fondo de Cultura Económica; 1998.

11. Conill J. Nueva propuesta de reconstrucción de las bases éticas del enfoque de las capacidades de Amartya Sen. 1-7. Disponible en: http:// www.unipv.it/deontica/ca2004/papers/conill.pdf

12. Delgado P, Salcedo T. Aspectos conceptuales sobre los indicadores de calidad de vida.1-31. Disponible en: http://www.webpondo.org/ files_oct_dic_03/ AspectosConceptuales.pdf

13. Saugstad A. La filosofía y la buena vida. PerSe [Internet] 2007; 2(8): 4-6. Disponible en: http://www.filosofia.com.mx/archivos/perse08.pdf

14. Fascioli A. Capacidades, perfeccionismo y autonomía del sujeto en el pensamiento de Amartya Sen. Actio 2012: 69-88. Disponible en: http:// www.fhuce.edu.uy/ public/actio/Textos/II-2/Fascioli.pdf

15. Pereira G. Fundamentación universalista de una lista de capacidades requerida por la justicia global. Diánoia 2012; 57: 79-102. Disponible en: http://dianoia.filosoficas. unam.mx/info/2006/ d57-Pereira.pdf

16. Rivero C. ¿Calidad de Vida en América Latina? Las reflexiones epistemológicas y teóricas que acompañan los procesos. Caracas: La Galaxia; 2000.

17. Maslow A. Una teoría de la motivación humana, 1943. Revisión psicológica. 2001; 50: 370-496. Disponible en: http://psychclassics.yorku.ca/ Maslow/motivation.htm

18. Palacios D, Castro C, Reygadas D. Calidad de Vida: una perspectiva individual. Disponible en: http://www.monografias.com/trabajos15/ calidad-de-vida/calidad-de-vida.shtml 